\title{
Review
}

\section{Three-dimensional exploration of the solar wind using observations of interplanetary scintillation}

\author{
By Munetoshi TOKUMARU*1, $\dagger$
}

(Communicated by Atsuhiro NishidA, M.J.A.)

\begin{abstract}
The solar wind, a supersonic plasma flow continuously emanating from the Sun, governs the space environment in a vast region extending to the boundary of the heliosphere ( $\sim 100 \mathrm{AU})$. Precise understanding of the solar wind is of importance not only because it will satisfy scientific interest in an enigmatic astrophysical phenomenon, but because it has broad impacts on relevant fields. Interplanetary scintillation (IPS) of compact radio sources at meter to centimeter wavelengths serves as a useful ground-based method for investigating the solar wind. IPS measurements of the solar wind at a frequency of $327 \mathrm{MHz}$ have been carried out regularly since the 1980s using the multi-station system of the Solar-Terrestrial Environment Laboratory (STEL) of Nagoya University. This paper reviews new aspects of the solar wind revealed from our IPS observations.
\end{abstract}

Keywords: the Sun, solar wind, interplanetary scintillation, heliosphere

\section{Introduction}

The Sun's upper atmosphere is continually expanding into interplanetary space to form the solar wind, which is a hot, tenuous supersonic outflow of magnetized plasma with a speed of $300 \sim 750 \mathrm{~km} / \mathrm{s}$. The solar wind dominates a vast region around the Sun, and creates a bubble in the interstellar medium known as the heliosphere. The boundary of the heliosphere is expected to be at $\sim 100 \mathrm{AU}$ from the Sun, therefore all solar system planets are engulfed in the solar wind. The existence of the solar wind was theoretically modeled in $1958,{ }^{1)}$ and verified by direct measurements with Luna 2 in 1959.2) Since then, basic properties of the solar wind have been extensively investigated using direct (in situ) and indirect (remote sensing) methods. Despite the wealth of observational data compiled so far, many fundamental questions concerning the solar wind remain unsettled. The origin and acceleration mechanism of the solar wind is the most enigmatic issue

*1 Solar-Terrestrial Environment Laboratory, Nagoya University, Aichi, Japan.

$\dagger$ Correspondence should be addressed: M. Tokumaru, Solar-Terrestrial Environment Laboratory, Nagoya University, Furo-cho, Chikusa-ku, Nagoya, Aichi 464-8601, Japan (e-mail: tokumaru@stelab.nagoya-u.ac.jp). among them, and there is no established theory which fully accounts for the formation of the solar wind. A striking feature revealed from observations is that the solar wind ceaselessly varies on a wide range of spatial and temporal scales. This enormous variability of the solar wind is known to deeply affect the Earth's upper atmosphere and space environment, sometimes resulting in serious damage on spaceborne and ground-based systems. ${ }^{3), 4)}$ Therefore, reliable predictions of the solar wind condition are required for maintaining today's technology-dependent society, and the improvement of our understanding of the solar wind is important not only from the viewpoint of space plasma physics but also from the viewpoint of its influence on the Earth's environment.

Remote-sensing observations of the solar wind have been conducted for more than 3 decades at the Solar-Terrestrial Environment Laboratory (STEL) of Nagoya University, using the interplanetary scintillation (IPS) method.5),6) This project aims to elucidate the enigmas of the solar wind from observations of its 3-dimensional properties, which are inaccessible with in situ measurements. Various important aspects of the solar wind have been unveiled from STEL IPS observations. The present paper provides a brief review of those results. Here, 
Table 1. Specifications and performance of the IPS antenna at each station

\begin{tabular}{|c|c|c|c|c|c|c|c|}
\hline \multirow[t]{2}{*}{ Station } & \multicolumn{2}{|c|}{$\begin{array}{l}\text { Aperture Size } \\
(\mathrm{m})\end{array}$} & \multirow{2}{*}{$\begin{array}{l}\text { Effective } \\
\text { Aperture } \\
\text { Area }\left(\mathrm{m}^{2}\right)\end{array}$} & \multicolumn{2}{|c|}{ Directivity Control } & \multirow{2}{*}{$\begin{array}{l}\text { No. } \\
\text { Elements }\end{array}$} & \multirow{2}{*}{$\begin{array}{c}\text { System } \\
\text { Temperature } \\
(\mathrm{K})\end{array}$} \\
\hline & $\mathrm{N}-\mathrm{S}$ & E-W & & $\mathrm{N}-\mathrm{S}$ & $\mathrm{E}-\mathrm{W}$ & & \\
\hline Toyokawa & 88 & 38 & 1970 & Electronically & Fixed & 192 & 146 \\
\hline Fuji & 20 & 100 & 1500 & Mechanically & Electronically & 192 & 151 \\
\hline Sugadaira & 20 & 100 & 1120 & Mechanically & Electronically & 192 & 229 \\
\hline Kiso & 27 & 73.5 & 1409 & Mechanically & Electronically & 144 & 221 \\
\hline
\end{tabular}

I focus on recent results which have been obtained from IPS studies after the mid-1990s, when improved observations with innovative methods for data analysis became available at STEL. The outline of this paper is as follows: section 2 describes the STEL multi-station IPS system dedicated to solar wind observations. Section 3 describes the 3-dimensional solar wind structure revealed from the computerassisted tomography (CAT) analysis of STEL IPS data. Sections 4 and 5 address the origin and acceleration mechanism of the solar wind using a combined analysis of IPS and coronal magnetic field data. Sections 6 and 7 show the short-term and longterm evolution of the solar wind as disclosed from STEL IPS observations. The summary is given in section 8 .

\section{IPS observations with $327-\mathrm{MHz}$ multi-station system}

Small-scale $(\sim 100 \mathrm{~km})$ electron density irregularities in the solar wind diffract radio waves from a celestial source with a compact diameter (usually less than 1 arc second), producing random fluctuations in the wave intensity observed on the ground with a time scale of 1 second or shorter. This phenomenon is called interplanetary scintillation (IPS), and has long been used since its discovery as an effective tool for probing the solar wind plasma. ${ }^{7), 8)}$ The flow velocities have been derived from simultaneous observations of IPS at multiple stations under the assumption that density irregularities in the solar wind drift at the speed of the bulk motion (the so-called frozen-in hypothesis).5),9),10) The large-scale density distribution in the solar wind has been determined from IPS observations, since density fluctuations $\left(\Delta N_{e}\right)$ are approximately proportional to the density. ${ }^{11)}$ The important point to note is that IPS observations provide global information on the solar wind if many radio sources are used. ${ }^{12), 13)}$ Furthermore, IPS observations provide not only macroscopic information of the solar wind plasma, but also reveal its microscopic properties such as the spectral shape and anisotropy of density turbulence, and its dissipation process. ${ }^{14)-16)}$

Multi-station IPS measurements of the solar wind have been conducted regularly over more than three decades at the Solar-Terrestrial Environment Laboratory (STEL) of Nagoya University using the UHF radio-telescope array, which is composed of large antennas at Toyokawa, Fuji, Sugadaira, and Kiso $^{6), 17)}$ (see Table 1). The locations of the antenna sites, which are shown in Fig. 1, were selected to form a set of baselines comparable to the Fresnel scale $(\sim 150 \mathrm{~km}$ for $327 \mathrm{MHz})$. Such a baseline configuration yields a good correlation between IPS data at the separated stations for any flow directions. The observation frequency of $327 \mathrm{MHz}$ (wavelength: $92 \mathrm{~cm}$ ) is optimal for surveying the inner heliosphere, since it enables us to probe the solar wind plasma between $\sim 0.1 \mathrm{AU}$ and $1 \mathrm{AU}$. Figure 2 shows the IPS antenna at Fuji, having a large-aperture asymmetric parabolic cylinder reflector. The dimension of the reflector is $100 \mathrm{~m}$ in east-west and $20 \mathrm{~m}$ in northsouth. A low-noise phased array receiver installed on the Fuji antenna enables observation with IPS for 30-40 scintillating sources a day. Specifications of IPS antennas at Sugadaira and Kiso are basically the same as those at Fuji, although there are many minor differences among them. STEL IPS observations at $327 \mathrm{MHz}$ began in 1983 with three stations at Toyokawa, Fuji, and Sugadaira, and the IPS antenna at Kiso was added to the observation system in 1993. Four-station IPS observations, which provide more reliable estimates of the solar wind speed, had been carried out until 2006, when the Toyokawa antenna was closed in order to upgrade it to a larger system with a higher sensitivity. Figure 3 shows the IPS antenna at Toyokawa, which is called the Solar Wind Imaging Facility (SWIFT). ${ }^{18)}$ This antenna, a median transit radio telescope with a pair of asymmetrical parabolic cylinder reflectors and a 192 elements phased array receiver, has the best 


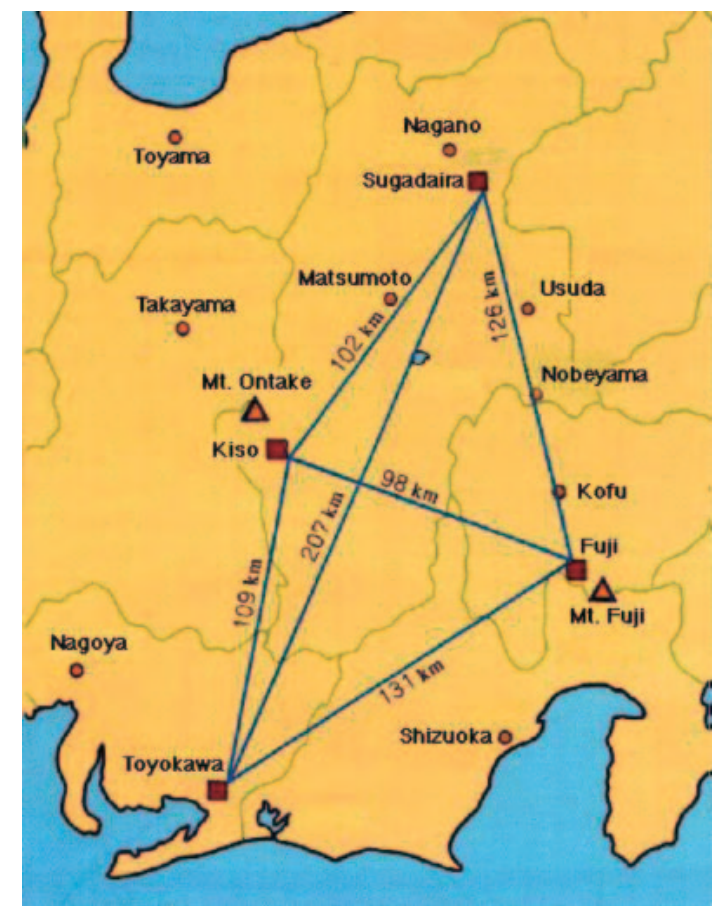

Fig. 1. Geographic locations of STEL IPS observatories.

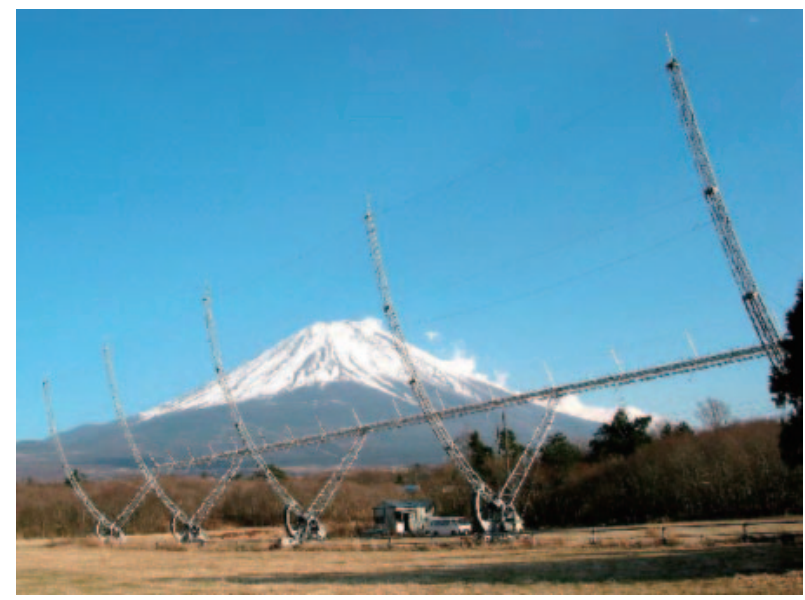

Fig. 2. The 327-MHz radiotelescope at Fuji Observatory.

performance of our four antennas (see Table 1). IPS observations with SWIFT began in 2008, and multi-station IPS data collected simultaneously with SWIFT were available starting in 2010. Note that IPS data at Toyokawa (i.e., SWIFT) are collected on a daily basis throughout the year, whereas those at other stations are interrupted during winter because of heavy snowfall. The solar wind speeds are derived from a cross-correlation analysis of multi-station IPS data, and the scintillation levels are calculated from

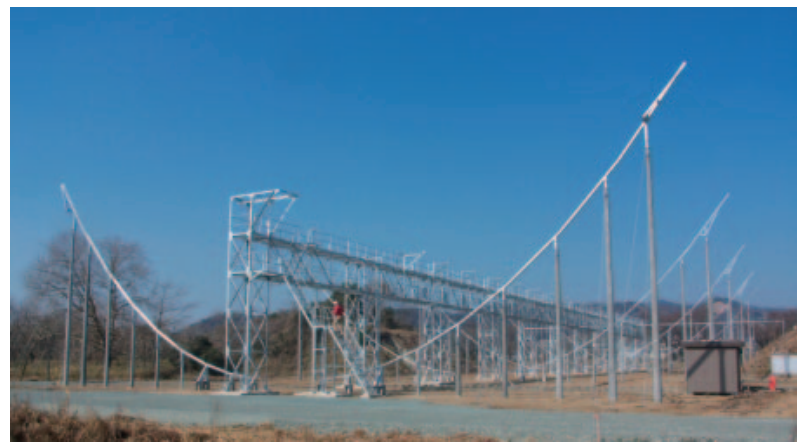

Fig. 3. The 327-MHz radiotelescope (Solar Wind Imaging Facility $^{19)}$ ) at Toyokawa Observatory.

the power spectra of IPS data at a given station. Those IPS data are freely available on the internet via our web site (http://stsw1.stelab.nagoya-u.ac.jp).

\section{Tomographic reconstruction of 3D solar wind structure}

Although IPS observations provide global information of the solar wind, it must be kept in mind that they are a line-of-sight (LOS) integration of the actual values obtained through the 3D solar wind structure. This LOS integration effect is known to bias IPS data and to blur fine features. We have developed the computer-assisted tomography (CAT) method to deconvolve LOS integration of IPS data and to retrieve the intrinsic solar wind distribution. ${ }^{19)-22)}$ At present, various versions of the IPS CAT method are available, including the multi-step CAT ${ }^{23)}$ two-reference-sphere CAT, ${ }^{24)}$ time-sequence CAT ${ }^{25)}$ MHD-IPS tomography, ${ }^{26)}$ and time-dependent tomography. ${ }^{27)}$

Figure 4 demonstrates how the IPS CAT method can retrieve the solar wind structure in the synoptic map. The upper map is derived from STEL IPS observations for Carrington rotations (CRs) 1909-1913 (April to September 1996), using the classical technique in which the point closest to the Sun on a line-of-sight is used as a reference point of the data (the so-called P-point assumption). In this map, solar wind speeds at high latitudes are lower than $700 \mathrm{~km} / \mathrm{s}$, inconsistent with those measured by Ulysses. This discrepancy is ascribed to the LOS integration effect. The lower map presents the result of the CAT analysis for the same IPS data as used in the upper map. We find from the map that the solar wind emanating from the high latitude region has speeds faster than $700 \mathrm{~km} / \mathrm{s}$, and that sharp boundaries exist between polar fast and equatorial slow winds. These features are consistent with Ulysses 
observations, suggesting that the LOS integration effect is removed by the CAT method. Figure 5 displays a direct comparison between Ulysses in situ measurements and the CAT analysis of IPS observations for CRs 1894-1896, corresponding to the period from April to June 1995 (blue dashed line). ${ }^{23)}$ The solar wind speeds from Ulysses and IPS measurements are plotted as a function of heliographic latitude in the figure. It is clearly revealed from this figure that the solar wind is composed of high- and low-speed streams distributed over the poles and equator, respectively, and that the IPS data are in a good agreement with in situ measurements by Ulysses for the period of September 1994 to July 1995 (red dashed line). ${ }^{28)}$ Thus, the CAT analysis of IPS observations provides global data of the solar wind that are as reliable as in situ measurements. Moreover, it may be concluded that the velocity gradient and north-south asymmetry found in the polar fast wind by Ulysses represent intrinsically stable structures, not temporal changes, since they are consistent with IPS observations taken over a much shorter period than the Ulysses poleto-pole fast scan (10 months). These structures are considered to reflect the dependence of the solar wind speed on the magnetic field configuration at the source region.

\section{Sources of the solar wind}

The source region of the solar wind can be identified by tracing the coronal magnetic field line from the photosphere to the source surface, since the corona is dominated by a low- $\beta$ plasma. Figure 6 demonstrates the coronal magnetic field lines computed from magnetograph observations at Kitt Peak using the potential field model. ${ }^{29)}$ The source surface map of the solar wind speed derived from STEL IPS observations is also indicated in the upper plot of the figure. It is clearly shown here that the fast wind at high latitudes is closely associated with polar coronal holes where the magnetic field is open, and also that the slow wind at the equator is mostly linked to either the boundary region of polar coronal holes or the isolated low-latitude coronal holes.

What we have discovered from a detailed comparison between coronal magnetic fields and IPS observations is that there is another source region for solar winds with a very low speed such as $<350 \mathrm{~km} / \mathrm{s.}^{30)}$ Figure 7 shows magnetic potentialfield lines calculated from Kitt Peak observations, and the solar wind speed map on the source surface from STEL IPS observations. In this figure, open field lines corresponding to the very low speed region originate from the vicinity of the closed loops (active regions). Thus, the source region of the very low speed wind is located in the vicinity of the active regions, consistent with plasma outflows observed by Hinode X-ray imaging. ${ }^{31)}$ The very low speed of this solar wind is considered to be a consequence of rapid areal divergence of the magnetic flux tube at a rate faster than radial expansion. The very low speed wind is found to have different characteristics of helium abundance and variances in solar wind properties, which distinguishes it from other regions of slow solar winds. ${ }^{32)}$ Our IPS observations show that the solar wind density fluctuation level $\Delta N_{e}$ for $V<350 \mathrm{~km} / \mathrm{s}$ tends to drop below the empirical relation $\Delta N_{e} \propto V^{-0.5}$, where $V$ is the solar wind speed. ${ }^{33)}$ The abnormally-low density $\left(\sim 0.1 \mathrm{~cm}^{-3}\right)$ plasma observed in 1999 and 2002 was associated with very low speed flow from a source located in the vicinity of an active region. ${ }^{34), 35)}$ These facts may be ascribed to the different source conditions of this solar wind. Similar low-speed winds have been identified from the CAT analysis of our IPS observations over the pole at solar maximum, when the polar coronal hole shrinks in size before and after its disappearance. ${ }^{36), 37)}$ This polar low-speed wind is associated with a super radially expanding field into interplanetary space, as is the case of the activeregion associated low-speed wind. Thus, the configuration of the coronal magnetic field plays an important role in determining the solar wind acceleration (further discussion in the next section). A scenario to systematically account for the relation between the solar wind and its source region has been proposed under the hypothesis of the fluxdivergence-dependent acceleration model. ${ }^{32)}$

\section{Acceleration mechanism of the solar wind}

While the detailed mechanism that accelerates the solar wind remains elusive, it is certain that the Sun's magnetic field plays an important role in it. ${ }^{38)}$ An inverse relationship has been reported to exist between the terminal solar wind speed $V$ and the areal expansion rate $f$ of magnetic flux tubes from earlier surveys; i.e., $V \propto 1 / f^{29), 39), 40)}$ We found an empirical relationship which connects $\mathrm{V}$ and the physical property of the coronal magnetic field more closely. ${ }^{41), 42)}$ Figure 8 shows a correlation between $V$ and $B / f$, where $B$ is the photospheric magnetic field intensity. The solar wind speed data are obtained from STEL IPS observations, and the parameter $B / f$ is from the calculation of a potential field using Kitt 


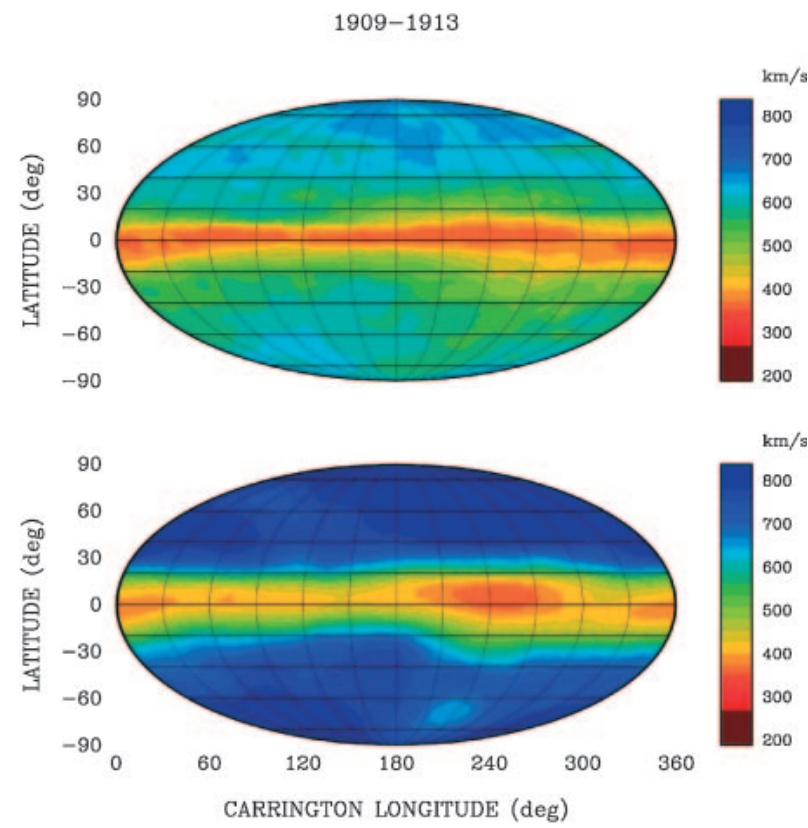

Fig. 4. (upper panel) Synoptic map of solar wind speed on source surface derived from STEL IPS observations during CRs 19091913 using the classical technique, and (lower panel) derived using CAT analysis.

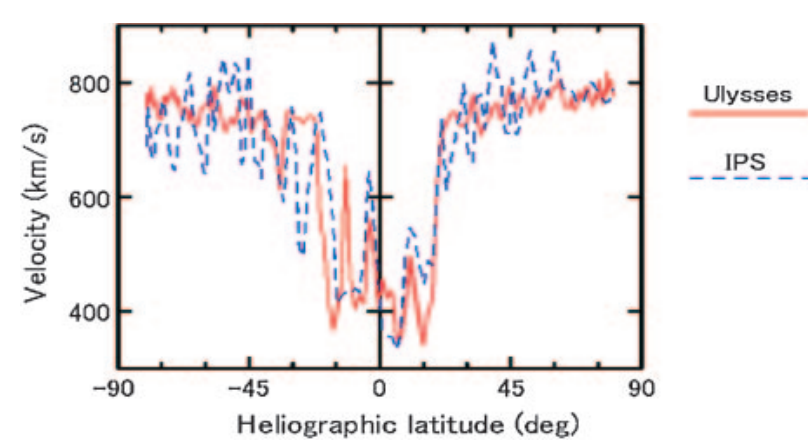

Fig. 5. Comparison of latitudinal solar wind speed structures between Ulysses observations (solid red line) and IPS CAT analysis (dashed blue line). IPS CAT speed data are sampled along Ulysses trajectory. This figure is adopted from Kojima et al. $(2001){ }^{23)}$

Peak magnetograph observations. A high degree of positive correlation is demonstrated between $V$ and $B / f$. Moreover, we found that this correlation is better than that between $\mathrm{V}$ and $1 / f$. Our result provides an important implication concerning the solar wind acceleration mechanism. A theoretical explanation for $V \propto B / f$ has been given using the Alfven wave driven solar wind model. ${ }^{43)}$

The radial dependence of the solar wind speed also provides important information for gaining

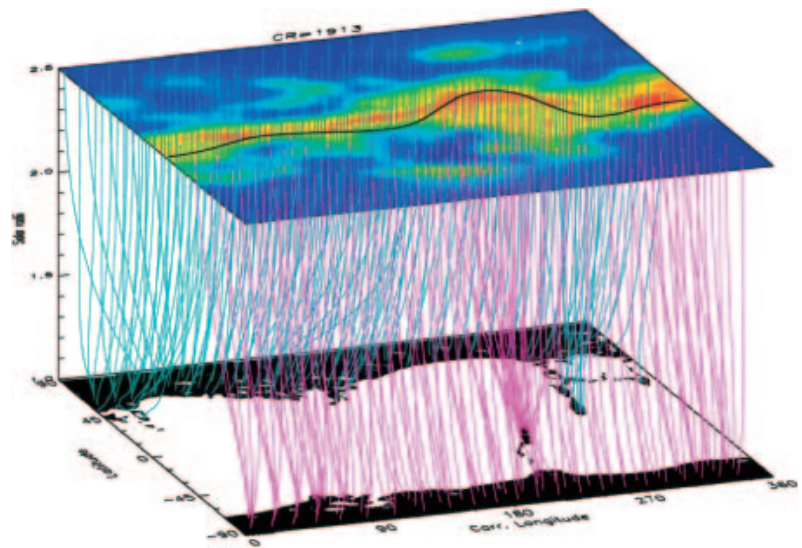

Fig. 6. (upper plot) Solar wind speed map on source surface at 2.5 Rs from CAT analysis of STEL IPS observations, and (lower plot) coronal hole map on photosphere, and (blue and red solid lines) open magnetic potential-field lines between photosphere and source surface. Data are for CR 1913, and field lines are calculated from Kitt Peak magnetograph observations. In the solar wind speed map, the color scale is the same as Fig. 4. In the lower plot, black areas indicate the coronal holes where field lines open into interplanetary space.

\section{CARRINGTON ROTATION NUMBER : 1913}

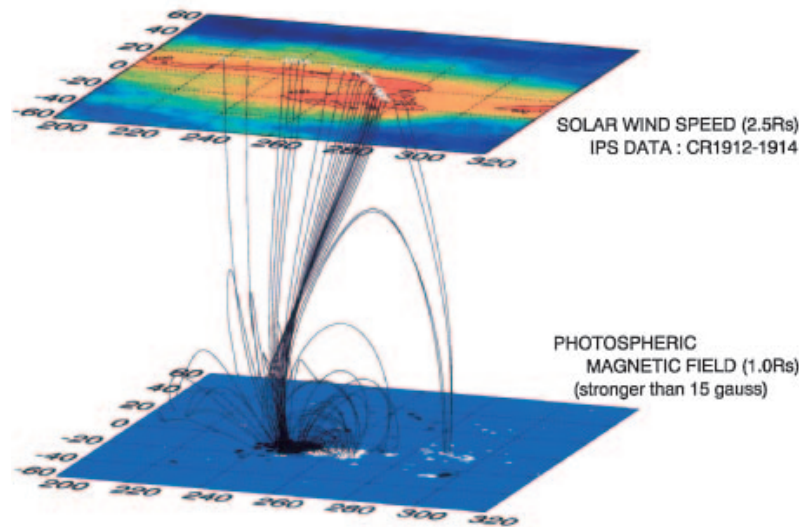

Fig. 7. Magnetic field lines from a potential field calculation shown from photosphere to source surface. Data are for CR 1913. Field lines connecting photosphere and source surface are shown for an expansion factor $>2000$, and closed loops in the corona are shown for field lines with photospheric magnetic fields stronger than $15 \mathrm{G}$. On the source surface, red areas bounded by black lines show regions where speeds $<400 \mathrm{~km} / \mathrm{s}$, and white spots are locations with a flux expansion factor $>2000$. This figure is adopted from Kojima et al. (1999). ${ }^{30)}$

insight into the solar wind acceleration mechanism. We have investigated the radial gradient of the speed of high-latitude fast solar wind in the distance range of 0.13-0.9 AU using the two-reference sphere CAT analysis of STEL IPS observations. ${ }^{24)}$ As a result, 


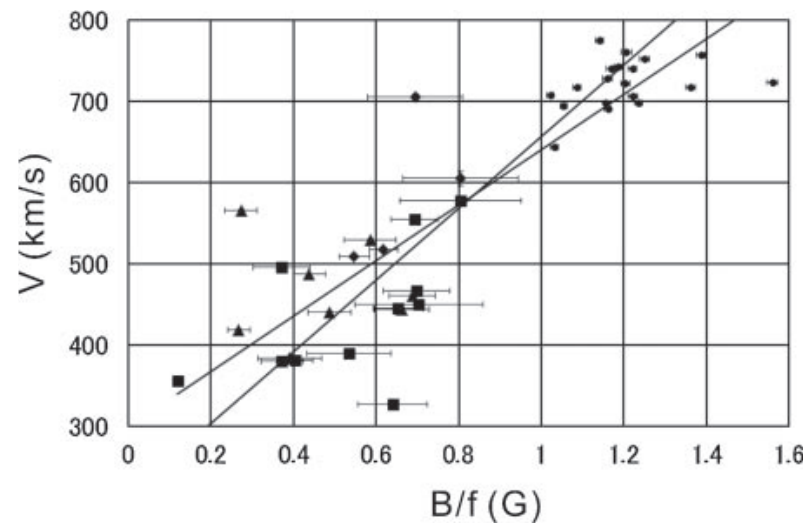

Fig. 8. Correlation diagram between $\mathrm{V}$ and $B / f$. Triangles represent the equatorial coronal holes associated with active regions with a large flux expansion rate and relatively strong magnetic field. Squares represent the isolated mid-latitude coronal holes with a medium expansion rate and weaker magnetic field. Dots represent the polar coronal holes with a smaller expansion rate and weaker magnetic field, and diamonds represent the equatorial extension of polar coronal holes. Two regression lines are for $\mathrm{V}$ from $B / f$ and for $B / f$ from $\mathrm{V}$, respectively. The correlation coefficient is 0.88 . The figure is adopted from Kojima et al. (2004). ${ }^{41)}$

a small but measurable increase in the solar wind speed, $19 \pm 17 \mathrm{~km} / \mathrm{s}$, is detected between 0.13 $0.3 \mathrm{AU}$ and $0.3-0.9 \mathrm{AU}$. This result suggests that the fast wind is accelerated almost to its terminal speed within $20 \mathrm{Rs}$, but that the acceleration is not fully complete at this solar distance, and continues beyond 30 Rs.

\section{3D Properties of interplanetary disturbances associated with CMEs}

The coronal mass ejection (CME) is known as a solar driver of significant disturbances in the interplanetary and near-Earth space environments. ${ }^{44), 45)}$ Since these disturbances cause hazardous conditions for space systems and ground-based facilities, reliable predictions of a CME's arrival at Earth and its magnitude are needed to reduce their risks. ${ }^{3)}$ However, our current understanding of $\mathrm{CME}$ and its interplanetary counterpart (ICME) is insufficient for making reliable space weather predictions. In particular, the 3D structure and radial evolution of ICMEs are poorly understood, owing to the shortage of global observations for transient solar wind features. IPS serves as a useful tool for studying 3D properties of ICMEs, since it can produce a sky map of the solar wind on a daily basis if one collects data for many lines-of-sight regularly. ${ }^{12)}$ Figure 9 (right) and (left) shows a sky projection map of g-values derived from
STEL IPS observations and the LASCO C3 coronagraph image, respectively, for the CME event on July $10,2000{ }^{46)}$ The g-value, which is given by $\mathrm{g}=\Delta S \overline{\Delta S(\varepsilon)}$ (where $\Delta S$ is the observed scintillation level and $\overline{\Delta S(\varepsilon)}$ is the mean level for the source at solar elongation $\varepsilon$ ), represents relative variation of $\Delta N_{e}$ along a LOS, and it abruptly enhances when highly turbulent plasma associated with an ICME crosses the LOS. As shown in the figure, a bright CME was observed with LASCO on the northeast limb of the Sun on July 10, 2000, and clear enhancements in g-value $(\mathrm{g}>1.5)$ were detected in the northeast quadrant of the Sun-centered sky plane from STEL IPS observations made about one day after the CME occurrence. The g-value enhancements form an arc-shaped structure, which matches well with a radial extension of the LASCO CME image. The arc-shaped g-value enhancements can be explained by compressed plasma associated with the leading edge of a bubble-shaped ICME travelling between the Sun and the Earth's orbit. Thus, the sky-plane distribution of g-value enhancements provides useful information for inferring the 3D morphology of the ICME, and the variety of enhanced g-value distribution reflects differences in the ICME structure. ${ }^{47), 48)}$

We have reconstructed the intrinsic 3D form of an ICME from a deconvolution analysis of g-value data. In this analysis, a kinematic model of an ICME is fit to IPS observations by calculating simulated g-values from LOS integration. ${ }^{49), 50)}$ The $3 \mathrm{D}$ reconstructions of the ICME have been compared with in situ and white-light measurements. ${ }^{51), 52)}$ An interesting feature revealed from this analysis is a loopshaped high- $\Delta N_{e}$ structure, which is often observed in Earth-directed (so-called "halo"53) CME events. Figure 10 shows a sky projection map of g-values observed for the July 14, 2000 halo CME event, and the loop-shaped structure reconstructed with the model fitting analysis. ${ }^{49)}$ These reconstructions are shown to approximately follow the time-dependent reconstructions from the UCSD group. ${ }^{54), 55)}$ The axis orientation of the loop-shaped structure was found to be generally consistent with that of the magnetic flux rope associated with the ICME. ${ }^{49), 52), 56)}$ This fact suggests that this structure may be formed through the interaction between the magnetic flux rope and the background solar wind; i.e., its origin is the compressed ambient solar wind. Nevertheless, observational evidence showing another origin for the loop-shaped structure has been reported from simultaneous IPS and white-light observations of the 

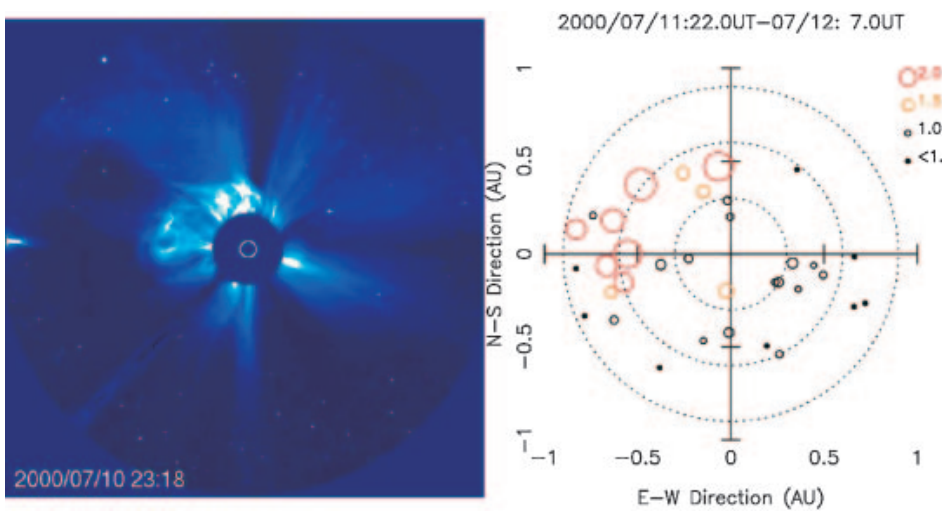

Fig. 9. (left) SOHO/LASCO C3 image of the CME on July 10, 2000, and (right) all-sky map of g-value obtained from STEL IPS observations for July 11 22h UT-July $127 \mathrm{~h}, 2000$. This figure is adopted from Tokumaru et al. (2003). ${ }^{46)}$
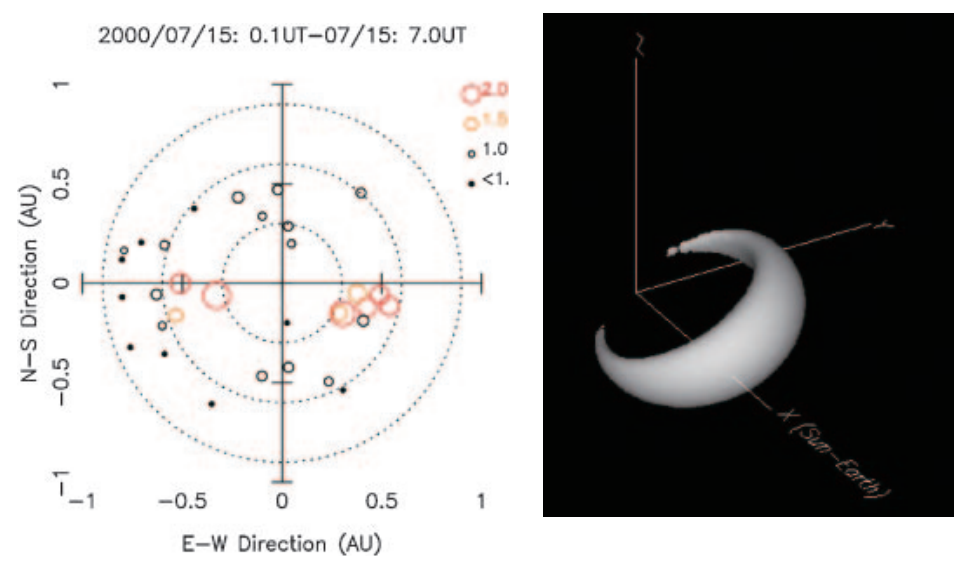

Fig. 10. (left) All-sky map of g-value obtained from STEL IPS observations on July 14, 2000, and (right) remote-observer's view of 3D $\Delta N_{e}$ distribution reconstructed from model fitting analysis. This figure is adopted from Tokumaru et al. (2003). ${ }^{49)}$

interplanetary disturbance associated with the intense halo CME event on October 28, 2003. ${ }^{52), 57)}$ The observations suggest that the high- $\Delta N_{e}$ loop observed in this event was more likely to represent the dense plasma ejected into the interplanetary medium from the corona rather than the compressed plasma of the interaction region. The high density within this loop is attributed to solar prominence associated with the CME eruption, suggesting that a portion of the magnetic flux rope contains dense coronal material. ${ }^{57)}$ Clearly these results provide important observational constraints for modeling the 3D ICME structure and its formation process.

STEL IPS observations have been used to study the radial evolution of CME speed during propagation, which is key knowledge for predicting space weather at Earth. Figure 11 exhibits the radial variation of CME speed for various events by combining data from LASCO, STEL IPS, and near-
Earth in situ measurements. ${ }^{58)}$ As is evident from this figure, fast (slow) CMEs decelerate (accelerate) asymptotically to the ambient solar wind speed as they propagate through the solar wind. The deceleration or acceleration rates depend on the speed difference between CME and the ambient solar wind, and steep gradients of the speed change occur near the Sun. This suggests that the drag force via the interaction with the solar wind plays a crucial role in determining the propagation speed of CMEs. However, it should be noted that the radial evolution of CME speeds shows a significantly different profile for some events. A combined study of IPS observations at STEL and Ooty (India) for the July 14, 2000 CME event revealed a two-step deceleration profile which is composed of a flat slope for $<0.5 \mathrm{AU}$ and a steeper fall for $>0.5 \mathrm{AU}^{59)}$ This implies that a driving agent acts on the CME travelling in the interplanetary medium to counterbalance the drag effect by 
the ambient solar wind. Although the physical process of this driving agent remains an open question, the toroidal Lorentz force by the magnetic flux rope ${ }^{60)}$ may be regarded as a possible underlying mechanism.

\section{Solar cycle variation of the solar wind structure}

Long-term IPS observations have shown that the $3 \mathrm{D}$ structure of the solar wind changes drastically with the solar activity cycle. Early studies using IPS observations in cycle 21 have revealed a close association of the slow wind with the large-scale magnetic neutral line and also disclosed the marked growth of fast solar winds over the poles when solar activity was low. ${ }^{61)-63)}$ Figure 12 demonstrates the evolution of the solar wind speed distribution derived from STEL IPS observations between 1991 and 2000; i.e., between the maxima of cycles 22 and 23. A systematic change of the speed distribution with the solar cycle is clearly revealed in this figure. We quantitatively investigated the solar cycle change of the solar wind structure during cycles 22 and 23 using STEL IPS observations. ${ }^{64)}$ Figure 13 demonstrates the year-by-year variation in the fractional area on the source surface corresponding to five different solar wind speed groups between 1985 and 2009 . CAT analysis is employed here to determine the distribution of the solar wind speed on the source surface. The upper, middle, and lower panels in the figure correspond to data for all latitudes, high latitudes $\left(\mid\right.$ lat $\left.\mid>70^{\circ}\right)$, and low latitudes $\left(\mid\right.$ lat $\left.\mid<10^{\circ}\right)$, respectively. A clear dependence on solar wind speed is apparent in IPS data for all latitudes (upper panel of Fig. 13). The fast (slow) wind area increases as the solar activity declines (rises), and reaches a peak at solar minimum (maximum). We note that fractional areas of the intermediate-speed solar wind remain mostly unchanged over two cycles. The response of the solar wind distribution to the solar cycle depends on the heliographic latitude. The high-latitude solar wind (middle panel of Fig. 13) is dominated by fast speed streams except for a few years around the solar maximum. In contrast, slow speed wind largely prevails at low latitudes during the entire period (lower panel of Fig. 13). This solar cycle dependence of the solar wind distribution is closely linked to the evolution of the Sun's large-scale magnetic field. Figure 14 shows correlations between polar magnetic fields, which represent the axial dipole moment of the Sun, and factional areas of fast (left) or slow (right) winds. The polar field data were obtained from magnetograph measurements at the Wilcox Solar Observatory, ${ }^{65)}$ and the yearly means of smoothed field strengths observed in the north and south are used to correlate with the IPS data. As shown in the figure, fast (slow) wind areas have a positive (negative) correlation with polar fields. The correlation coefficients for fast and slow winds are 0.74 and -0.65 , respectively. Such strong control of the polar fields on the solar wind distribution is considered to occur through the formation of coronal holes over the poles. The important point to note in this figure is that IPS data after 2005 follow a slightly different track in the correlation diagram. This may be ascribed to the peculiarity of the recent solar activity. ${ }^{66)}$ Many peculiar aspects of the solar activity have been reported for the last (cycle 23/24) minimum; i.e., an extremely long spotless condition, weaker polar field strength, and a significant reduction in the solar wind dynamic pressure have been observed in the last minimum. ${ }^{67)}$ Our IPS observations have revealed that the solar wind speed distribution at the last minimum is greatly different from that at the previous minimum. ${ }^{68)}$ As demonstrated in Fig. 13, low-latitude fast wind areas at the last minimum are significantly larger than those at the previous (cycle 22/23) minimum, and this is consistent with a prominent growth of equatorial coronal holes at the last minimum. ${ }^{69)}$ We also note that high-latitude fast wind areas at the last minimum are smaller than those in the previous minimum. ${ }^{64)}$ Another different feature revealed from our IPS observations is a gradual drop in the solar wind density turbulence level after the declining phase of cycle 23. ${ }^{13), 70)}$ This drop occurs globally, but is observed more conspicuously at low latitudes. This is consistent with a prominent growth of fast winds and coronal holes at low latitude for the last minimum, ${ }^{69)}$ and is also consistent with a reduction of the solar wind dynamic pressure ${ }^{67)}$ if $\Delta N_{e}$ is proportional to the solar wind bulk density. Our finding supports the speculation that the heliosphere is shrinking. ${ }^{67), 71)}$ In general, our IPS observations demonstrate that the $3 \mathrm{D}$ structure of the solar wind undergoes a drastic change in response to the peculiar solar dynamo activity.

\section{Summary}

The 3D properties of the solar wind, which are inaccessible to in situ measurements, have been investigated using our IPS observations and the CAT method. The close relation disclosed between the global solar wind distribution and coronal 
magnetic fields suggests that the coronal magnetic field plays a crucial role in accelerating the solar wind. We found that the very-low-speed $(V<$ $350 \mathrm{~km} / \mathrm{s}$ ) solar wind emanates from the open flux areas in the vicinity of active regions. This solar wind

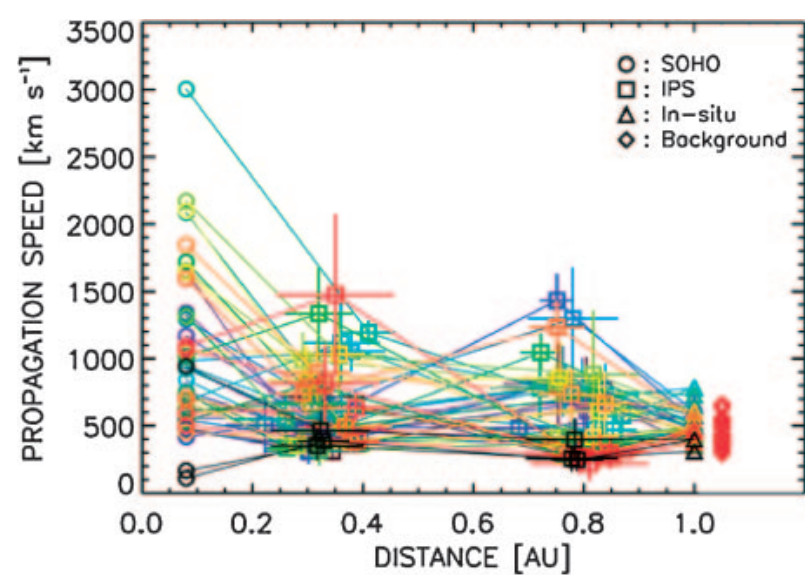

Fig. 11. Radial evolution of propagation speeds determined for 45 ICMEs. Open circles, squares, and triangles indicate speeds of ICMEs measured by SOHO/LASCO, IPS, and in situ observations, respectively. Open diamonds (red) indicate speeds of background solar wind measured in situ at $1 \mathrm{AU}$. The in situ speeds of the ICME and the background solar wind are determined as the average solar wind speeds over the ICME interval and between the CME occurrence and the arrival of the ICME leading edge at $1 \mathrm{AU}$, respectively. This figure is adopted from Iju et al. (2012). ${ }^{58)}$ source is characterized by extremely large superradial expansion of flux tubes. Such a magnetic field configuration is a key factor for producing very-lowspeed streams, and it may be responsible for the formation of rarefied plasma and reduced turbulence, although a further study is needed to confirm this. We also demonstrated that the polar fast solar wind almost reaches its terminal speed by $20 \mathrm{Rs}$, but a small acceleration continues beyond 30 Rs. This fact places an important observational constraint on

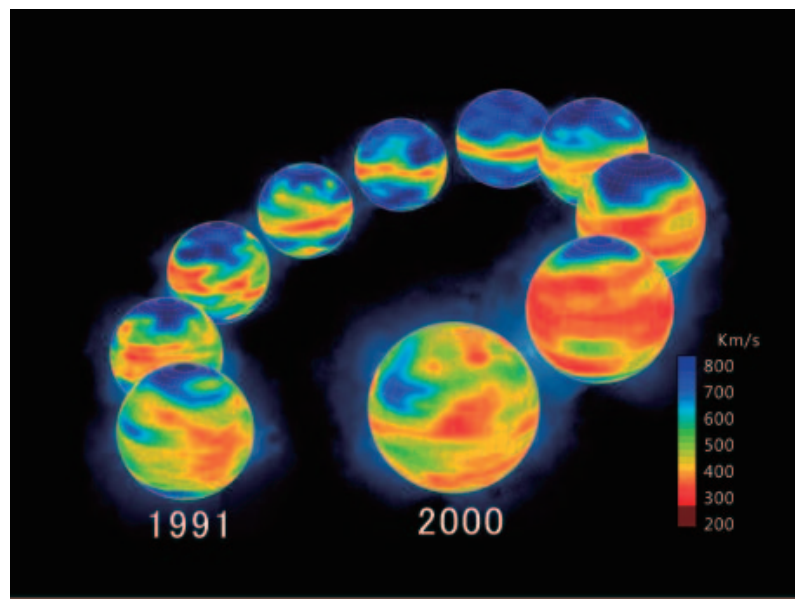

Fig. 12. Evolution of solar wind speed distribution from STEL IPS observations for the period from 1991 to 2000 (clockwise). A typical map is shown for each year.

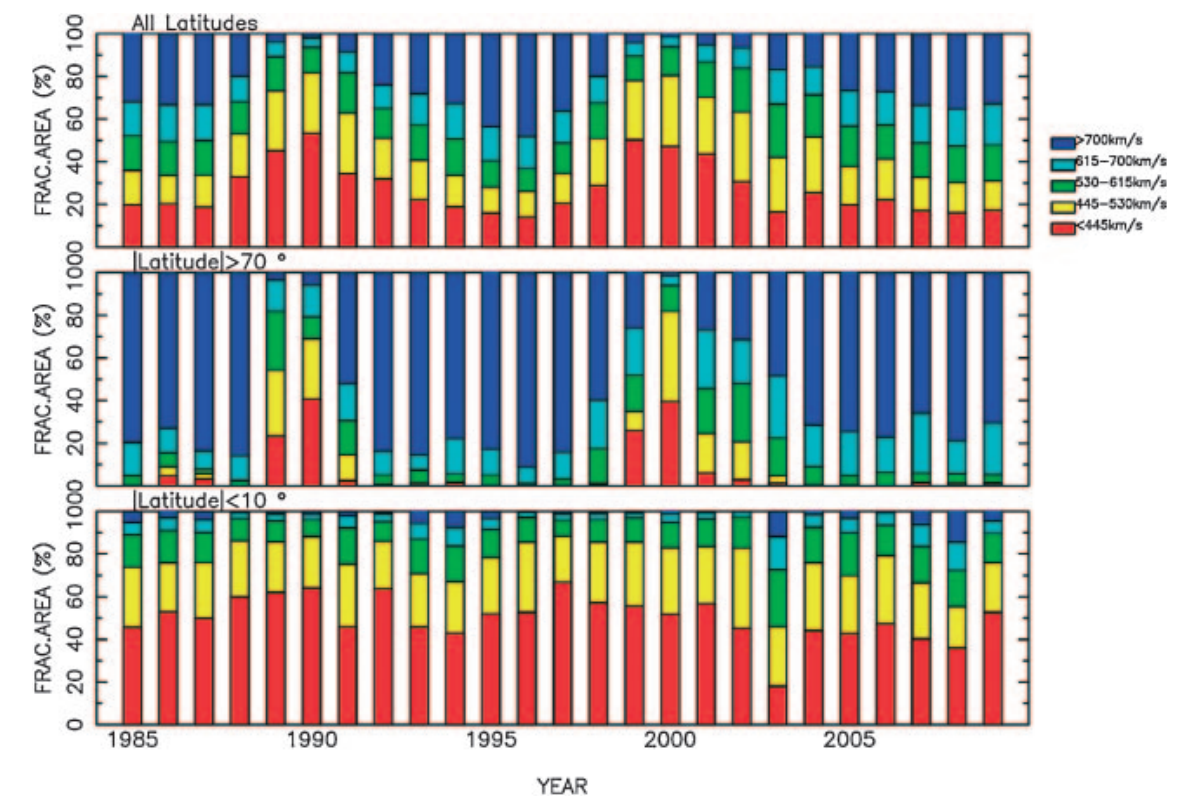

Fig. 13. Year-by-year variations in source surface areas corresponding to different solar wind speeds between 1985 and 2009 . Upper, middle, and lower panels correspond to all-latitude, high-latitude, and low-latitude data, respectively. 

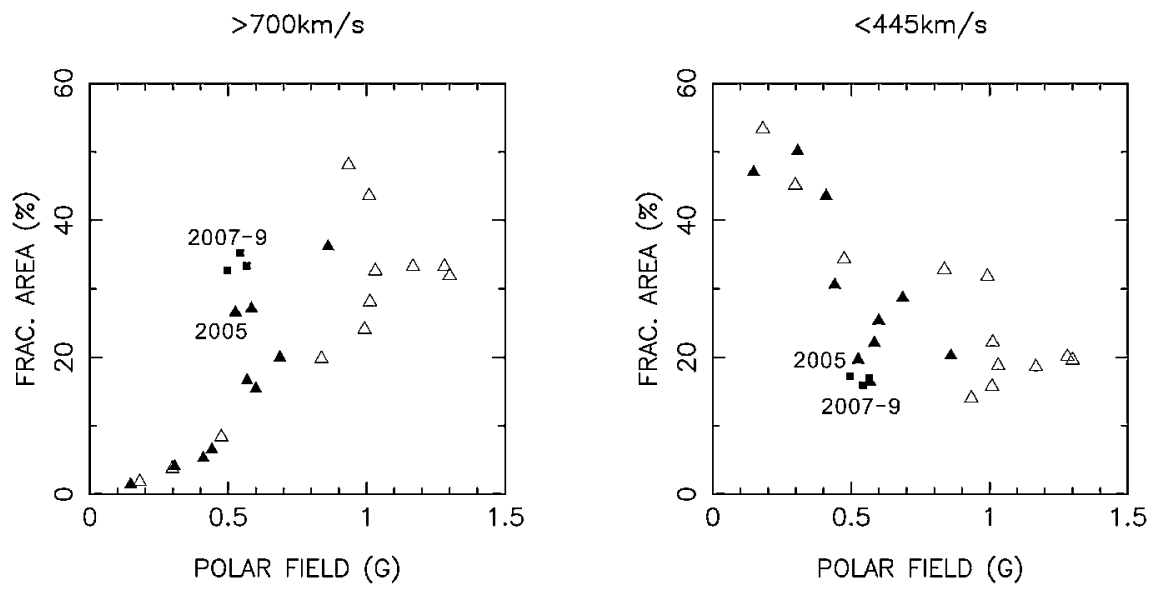

Fig. 14. Fractional areas versus yearly mean polar field strength for (left) $>700 \mathrm{~km} / \mathrm{s}$ and (right) $<445 \mathrm{~km} / \mathrm{s}$. Open and filled symbols represent data taken during 1985-1996 (cycle 22) and 1997-2009 (cycle 23), respectively. Filled squares indicate data taken during 2007-2009 (cycle 23/24 minimum).

theoretical models of the solar wind acceleration. The 3D morphology and propagation of ICMEs in the solar wind have been revealed from our IPS observations. The loop-shaped structure identified from our g-value data provides an important implication on the radial evolution of ICMEs. The deceleration or acceleration profiles of ICMEs observed between the Sun and 1 AU suggest that drag force via interaction with the ambient solar wind is a dominant agent for controlling their propagation. The two-step deceleration observed for an intense ICME event, on the other hand, may suggest that continuous injection of driving energy can occur. These results are useful for improving space weather predictions. Our IPS observations conducted over multiple cycles have clearly revealed that the global distribution of the solar wind undergoes a remarkable change with solar activity. This change is closely linked with polar field variation and is thus regarded as a manifestation of the solar dynamo activity. A significant difference in the global distribution of the solar wind exhibited in IPS observations between the last and previous minimum is considered to reflect the peculiar solar activity in the present cycle (cycle 24). Our IPS data suggest that a global reduction in the solar wind dynamic pressure is taking place in this cycle, consistent with the hypothesis of a shrinking heliosphere. This result provides valuable information for the exploration of the heliospheric boundary region, which is one of the cutting-edge research topics in space physics. Our view of the outer heliosphere is being revised by observations with Voyager $^{72)}$ and IBEX, ${ }^{73)}$ and our IPS observa- tions will be useful for developing this new view. Our IPS observations also provide important implications for understanding what happens in the Sun-Earth system during a greatly extended solar minimum. It is well-known that a cool climate occurred on the Earth during the prolonged spotless period in the 17 th century (the so-called Maunder minimum ${ }^{74)}$ ). While the physical process connecting low solar activity and Earth's cool climate is an open question, evolution of the global solar wind structure is assumed to play a crucial role. Solar activity is predicted to stay at low levels in the current and even forthcoming cycles. Hence, we need to continue collecting IPS data in order to elucidate further evolution of the global solar wind structure and its specific role in the Sun-Earth connection.

\section{Acknowledgements}

The IPS observations were carried out under the solar wind program of the Solar-Terrestrial Environment Laboratory (STEL) of Nagoya University. The author would like to thank the Wilcox Solar Observatory for providing open access to the magnetogram data. He also wishes to thank Masayoshi Kojima and two reviewers for providing valuable comments on this paper.

\section{References}

1) Parker, E.N. (1958) Dynamics of the interplanetary gas and magnetic field. Astrophys. J. 128, 664676.

2) Gringauz, K.I., Bezrukikh, V.V., Ozerov, V.D. and Rybchinskii, R.E. (1962) The study of interplan- 
etary ionized gas, high-energy electrons and corpuscular radiation of the sun, employing three-electrode charged particle traps of the second Soviet rocket. Planet. Space Sci. 9, 103-107.

3) Song, P., Singer, H.J. and Siscoe, G.L. (eds.) (2001) Space Weather. AGU monogram 125, American Geophysical Union, Washington, DC.

4) Moldwin, M. (2008) An Introduction to Space Weather. Cambridge University Press, Cambridge.

5) Kakinuma, T., Washimi, H. and Kojima, M. (1973) On the analysis of the observation of interplanetary scintillations observed with three spaced station. Publ. Astron. Soc. Jpn. 25, 271-280.

6) Asai, K., Ishida, Y., Kojima, M., Maruyama, K., Misawa, H. and Yoshimi, N. (1995) Multi-station system for solar wind observations using the interplanetary scintillation method. J. Geomag. Geoelectr. 47, 1107-1112.

7) Hewish, A., Scott, P.F. and Wills, D. (1964) Interplanetary scintillation of small diameter radio sources. Nature 203, 1214-1217.

8) Coles, W.A. (1978) Interplanetary scintillation. Space Sci. Rev. 21, 411-425.

9) Dennison, P.A. and Hewish, A. (1967) The solar wind outside the plane of the ecliptic. Nature $\mathbf{2 1 3}$ 343-346.

10) Armstrong, J.W. and Coles, W.A. (1972) Analysis of three-station interplanetary scintillation. J. Geophys. Res. 77, 4602-4610.

11) Coles, W.A., Harmon, J.K., Lazarus, A.J. and Sullivan, J.D. (1978) Comparison of $74-\mathrm{MHz}$ interplanetary scintillation and IMP 7 observations of the solar wind during 1973. J. Geophys. Res. 83, 3337-3341.

12) Gapper, G.R., Hewish, A., Purvis, A. and DuffettSmith, P.J. (1982) Observing interplanetary disturbances from the ground. Nature 296, 633-636.

13) Tokumaru, M., Kojima, M., Ishida, Y., Yokobe, A. and Ohmi, T. (2000) Large-scale structure of solar wind turbulence near solar activity minimum. Adv. Space Res. 25, 1943-1946.

14) Coles, W.A. and Harmon, J.K. (1978) Interplanetary scintillation measurements of the electron density power spectrum in the solar wind. J. Geophys. Res. 83, 1413-1420.

15) Kojima, M. (1979) Anisotropic structure of solar wind plasma irregularities observed with interplanetary scintillation. Publ. Astron. Soc. Jpn. 31, 231-249.

16) Chashei, I.V., Kojima, M. and Tokumaru, M. (2000) Anisotropy of magnetosonic turbulence in the solar wind between 0.1 and 0.4 AU. J. Geophys. Res. 105, 27401-27408.

17) Kojima, M., Ishida, Y., Maruyama, K. and Kakinuma, T. (1982) An observation system of interplanetary scintillation at UHF. Proc. Res. Inst. Atmospherics 29, 61-83.

18) Tokumaru, M., Kojima, M., Fujiki, K., Maruyama, M., Maruyama, Y., Ito, H. and Iju, T. (2011) A newly-developed UHF radiotelescope for interplanetary scintillation observations; Solar Wind Imaging Facility. Radio Sci. 46, RS0F02 (doi:10.1029/
2011RS004694).

19) Kojima, M., Tokumaru, M., Watanabe, H., Yokobe, A., Asai, K., Jackson, B.V. and Hick, P.L. (1998) Heliospheric tomography using interplanetary scintillation observations, II. Latitude and heliocentric distance dependence of solar wind structure at 0.1-1 AU. J. Geophys. Res. 103, 1981-1989.

20) Asai, K., Kojima, M., Tokumaru, M., Yokobe, A., Jackson, B.V., Hick, P.L. and Manoharan, P.K. (1998) Heliospheric tomography using interplanetary scintillation observations, 3. Correlation between speed and electron density fluctuations in the solar wind. J. Geophys. Res. 103, 19912001.

21) Jackson, B.V., Hick, P.L., Kojima, M. and Yokobe, A. (1998) Heliospheric tomography using interplanetary scintillation observations, 1 . Combined Nagoya and Cambridge data. J. Geophys. Res. 103, 12049-12067.

22) Kojima, M., Tokumaru, M., Fujiki, K., Hayashi, K. and Jackson, B.V. (2007) IPS tomographic observations of 3D solar wind structure. Astron. Astrophys. Trans. 26, 467-476.

23) Kojima, M., Fujiki, K., Ohmi, T., Tokumaru, M., Yokobe, A. and Hakamada, K. (2001) Latitudinal velocity structures up to the solar poles estimated from interplanetary scintillation tomography analysis. J. Geophys. Res. 106, 15677-15686.

24) Kojima, M., Breen, A.R., Fujiki, K., Hayashi, K., Ohmi, T. and Tokumaru, M. (2004) Fast solar wind after the rapid acceleration. J. Geophys. Res. 109, A04103 (doi:10.1029/2003JA010247).

25) Fujiki, K., Kojima, M., Tokumaru, M., Ohmi, T., Yokobe, A., Hayashi, K., McComas, D.J. and Elliott, H.A. (2003) How did the solar wind structure change around the solar maximum?From interplanetary scintillation observation. Ann. Geophys. 21, 1257-1261.

26) Hayashi, K., Kojima, M., Tokumaru, M. and Fujiki, K. (2003) MHD tomography using interplanetary scintillation measurement. J. Geophys. Res. 108, 1102 (doi:10.1029/2002JA009567).

27) Jackson, B.V., Hick, P.L., Buffington, A., Bisi, M.M., Clover, J.M. and Tokumaru, M. (2010) Solar Mass Ejection Imager (SMEI) and interplanetary scintillation (IPS) 3D-reconstructions of the inner heliosphere. Adv. Geosci. 21, 339-366.

28) Woch, J., Axford, W.I., Mall, U., Wilken, B., Livi, S., Geiss, J., Gloeckler, G. and Forsyth, R.J. (1997) SWICS/Ulysses observations: The three-dimensional structure of the heliosphere in the declining/minimum phase of the solar cycle. Geophys. Res. Lett. 24, 2885-2888.

29) Hakamada, K. and Kojima, M. (1999) Solar wind speed and expansion rate of the coronal magnetic field during Carrington rotation 1909. Sol. Phys. 187, 115-122.

30) Kojima, M., Fujiki, K., Ohmi, T., Tokumaru, M., Yokobe, A. and Hakamada, K. (1999) Low-speed solar wind from the vicinity of solar active regions. J. Geophys. Res. 104, 16993-17003.

31) Sakao, T., Kano, R., Narukage, N., Kotoku, J., 
Bando, T., Detuca, E.E., Lundquist, L.L., Tsuneta, S., Harra, L.K., Katsukawa, Y., Kubo, M., Hara, H., Matsuzaki, K., Shimojo, M., Bookbinder, J.A., Golub, L., Korreck, K.E., Su, Y., Shibasaki, K., Shimizu, T. and Nakatani, I. (2007) Continuous plasma outflows from the edge of a solar active region as a possible source of solar wind. Science 318, 1585-1588.

32) Ohmi, T., Kojima, M., Tokumaru, M., Fujiki, K. and Hakamada, K. (2004) Origin of the slow solar wind. Adv. Space Res. 33, 689-695.

33) Tokumaru, M., Kojima, M. and Fujiki, K. (2012) Long-term evolution in the global distribution of solar wind speed and density fluctuations for 19972009. J. Geophys. Res. 117, A06108 (doi:10.1029/ 2011ja017379).

34) Janardhan, P., Fujiki, K., Kojima, M., Tokumaru, M. and Hakamada, K. (2005) Resolving the enigmatic solar wind disappearance event of 11 May 1999. J. Geophys. Res. 110, A08101 (doi:10.1029/2004JA010535).

35) Janardhan, P., Fujiki, K., Sawant, H.S., Kojima, M., Hakamada, K. and Krishnam, R. (2008) Source regions of solar wind disappearance events. J. Geophys. Res. 113, A013102 (doi:10.1029/ 2007JA012608).

36) Ohmi, T., Kojima, M., Yokobe, A., Tokumaru, M., Fujiki, K. and Hakamada, K. (2001) Polar lowspeed solar wind at the solar activity maximum. J. Geophys. Res. 106, 24923-24936.

37) Ohmi, T., Kojima, M., Tokumaru, M., Fujiki, K., Hayashi, K. and Hakamada, K. (2003) Polar lowspeed solar wind reappeared at the solar activity maximum of cycle 23. Geophys. Res. Lett. 30, 1409 (doi:10.1029/2002GL016347).

38) Barnes, A. (1992) Acceleration of the solar wind. Rev. Geophys. 30, 43-55.

39) Wang, Y.-M. and Sheeley, N.R. Jr. (1990) Solar wind speed and coronal flux-tube expansion. Astrophys. J. 355, 726-733.

40) Hakamada, K., Kojima, M., Tokumaru, M., Ohmi, T., Yokobe, A. and Fujiki, K. (2002) Solar wind speed and expansion rate of the coronal magnetic field in solar maximum and minimum phases. Sol. Phys. 207, 173-185.

41) Kojima, M., Fujiki, K., Hirano, M., Tokumaru, M., Ohmi, T. and Hakamada, K. (2004) Solar Wind Properties from IPS observations. In The Sun And The Heliosphere As An Integrated System (eds. Giannina Poletto, G. and Suess, S.T.). Kluwer Academic Publishers, Dordrecht, pp. 147-178.

42) Kojima, M., Tokumaru, M., Fujiki, K., Itoh, H., Murakami, T. and Hakamada, K. (2007) What coronal parameters determine solar wind speed? In New Solar Physics with Solar-B Mission (eds. Shibata, K., Nagata, S. and Sakurai, T.). AIP Conf. Ser. 369, 549-555.

43) Suzuki, T.K. (2006) Forecasting solar wind speeds. Astrophys. J. 640, L75-L78.

44) Tang, F., Tsurutani, B., Gonzalez, W.D., Akasofu, S.-I. and Smith, E.J. (1989) Solar sources of interplanetary southward $\mathrm{Bz}$ events responsible for major magnetic storms (1978-1979). J. Geophys. Res. 94, 3535-3541.

45) Gosling, J.T. (1993) The solar flare myth. J. Geophys. Res. 98, 18937-18949.

46) Tokumaru, M., Kojima, M., Fujiki, K. and Yamashita, M. (2003) Global structure of interplanetary coronal mass ejections retrieved from the model fitting analysis of radio scintillation observations. Proc. of 10th International Conference on Solar Wind, AIP Conf. Proc. Ser. 679, 729-732.

47) Tokumaru, M., Kojima, M., Fujiki, K. and Yokobe, A. (2000) Three-dimensional propagation of interplanetary disturbances detected with radio scintillation measurements at $327 \mathrm{MHz}$. J. Geophys. Res. 105, 10435-10453.

48) Tokumaru, M., Kojima, M., Fujiki, K., Yamashita, M. and Baba, D. (2005) Interplanetary consequences caused by the extremely intense solar activity during October-November 2003. J. Geophys. Res. 110, A01109 (doi:10.1029/2004JA010656).

49) Tokumaru, M., Kojima, M., Fujiki, K., Yamashita, M. and Yokobe, A. (2003) Toroidal-shaped interplanetary disturbance associated with the halo coronal mass ejection event on July 14 , 2000. J. Geophys. Res. 108, 1220 (doi:10.1029/ 2002JA009574).

50) Tokumaru, M., Kojima, M., Fujiki, K. and Yamashita, M. (2006) Tracking heliospheric disturbances by interplanetary scintillation. Nonlinear Process. Geophys. 13, 329-338.

51) Tokumaru, M., Yamashita, M., Kojima, M., Fujiki, K. and Nakagawa, T. (2006) Reconstructed global feature of interplanetary disturbance for the fullhalo coronal mass ejection event on 1999 September 20. Adv. Space Res. 38, 547-551.

52) Tokumaru, M., Kojima, M., Fujiki, K., Yamashita, M. and Jackson, B.V. (2007) The source and propagation of the interplanetary disturbance associated with the full-halo coronal mass ejection on October 28, 2003. J. Geophys. Res. 112, A05106 (doi:10.1029/2006JA012043).

53) Howard, R.A., Michels, D.J., Sheeley, N.R. Jr. and Koomen, M.J. (1982) The observation of a coronal transient directed at Earth. Astrophys. J. 263, L101-L104.

54) Jackson, B.V., Hick, P.P., Buffington, A., Kojima, M., Tokumaru, M., Fujiki, K., Ohmi, T. and Yamashita, M. (2003) Time-dependent tomography of heliospheric features using interplanetary scintillation (IPS) remote-sensing observations, Solar Wind Ten, AIP Conf. Proc. Ser. 679, pp. $75-78$

55) Jackson, B.V. (2011) The 3D analysis of the heliosphere using interplanetary scintillation and Thomson-scattering observations. Adv. Geosci. 30, 69-91.

56) Tokumaru, M., Kojima, M., Fujiki, K., Munakata, K., Kuwabara, T. and Marubashi, K. (2010) Relation between loop-shaped interplanetary disturbances and the magnetic flux rope. Adv. Geosci. 21, 21-32.

57) Jackson, B.V., Buffington, A., Hick, P.P., Wang, X. 
and Webb, D. (2006) Preliminary three-dimensional analysis of the heliospheric response to the 28 October $2003 \mathrm{CME}$ using SMEI white-light observations. J. Geophys. Res. 111, A04S91 (doi:10.1029/2004JA010942).

58) Iju, T., Tokumaru, M. and Fujiki, K. (2012) Radial speed evolution of interplanetary coronal mass ejections during solar cycle 23. Submitted to Sol. Phys.

59) Manoharan, P.K., Tokumaru, M., Pick, M., Subramanian, P., Ipavich, F.M., Schenk, K., Kaiser, M.L., Lepping, R.P. and Vourlidas, A. (2001) Coronal mass ejection of July 14, 2000 Flare Event: Imaging from near-Sun to Earth environment. Astrophys. J. 559, 1180-1189.

60) Chen, J. (1989) Effects of toroidal forces in current loops embedded in a background plasma. Astrophys. J. 338, 453-470.

61) Coles, W.A., Rickett, B.J., Rumsey, V.H., Kaufman, J.J., Turley, D.G., Ananthakrishnan, S., Armstrong, J.W., Harmons, J.K., Scott, S.L. and Sime, D.G. (1980) Solar cycle changes in the polar solar wind. Nature 286, 239-241.

62) Kojima, M. and Kakinuma, T. (1987) Solar cycle evolution of solar wind speed structure between 1973 and 1985. J. Geophys. Res. 92, 7269-7279.

63) Kojima, M. and Kakinuma, T. (1990) Solar cycle dependence of global distribution of solar wind speed. Space Sci. Rev. 53, 173-222.

64) Tokumaru, M., Kojima, M. and Fujiki, K. (2010) Solar cycle evolution of the solar wind speed distribution from 1985 to 2008. J. Geophys. Res. 115, A04102 (doi:10.1029/2009JA014628).

65) http://wso.stanford.edu/Polar.html.

66) Russell, C.T., Luhmann, J.G. and Jian, L.K. (2010) How unprecedented a solar minimum. Rev. Geophys. 48, RG2004 (doi:10.1029/2009RG000316).

67) McComas, D.J., Ebert, R.W., Elliot, H.A., Goldstein, B.E., Gosling, J.T., Schwadron, N.A. and Skoug, R.M. (2008) Weaker solar wind from the polar coronal holes and the whole Sun. Geophys. Res. Lett. 35, L18103 (doi:10.1029/ 2008GL034896).

68) Tokumaru, M., Kojima, M., Fujiki, K. and Hayashi, K. (2009) Non-dipolar solar wind structure observed in the cycle 23/24 minimum. Geophys. Res. Lett. 36, L09101 (doi:10.1029/2009GL037461).

69) de Toma, G. (2011) Evolution of coronal holes and implications for high-speed solar wind during the minimum between cycles 23 and 24. Sol. Phys. 274, 195-217.

70) Janardhan, P., Bisoi, S.K., Ananthakrishnan, S., Tokumaru, M. and Fujiki, K. (2011) The prelude to the deep minimum between solar cycles 23 and 24: interplanetary scintillation signatures in the inner heliosphere. Geophys. Res. Lett. 38, L20108 (doi:10.1029/2011GL049227).

71) Webber, W.R. and Intriligator, D.S. (2011) Voyagers 1 and 2 in a shrunken and squashed heliosphere. J. Geophys. Res. 116, A06105 (doi:10.1029/ 2011JA016478).

72) Decker, R.B., Krimigis, S.M., Roelof, E.C. and Hill, M.E. (2012) No meridional plasma flow in the heliosheath transition region. Nature 489, 124127.

73) McComas, D.J., Bzowski, M., Frisch, P., Crew, G.B., Dayeh, M.A., DeMajistre, R., Funsten, H.O., Fuselier, S.A., Gruntman, M., Janzen, P., Kubiak, M.A., Livadiotis, G., Möbius, E., Reisenfeld, D.B. and Schwadron, N.A. (2010) Evolving outer heliosphere: Large-scale stability and time variations. J. Geophys. Res. 115, A09113 (doi:10.1029/2010JA015569).

74) Eddy, J.A. (1976) The Maunder minimum. Science 192, 1189-1202.

(Received Sep. 24, 2012; accepted Nov. 20, 2012)

\section{Profile}

Munetoshi Tokumaru was born in 1957, and received his M.S. in 1982 and Ph.D. in 1986 from the Geophysical Institute, Tohoku University. He started his research career in 1985 in the Radio Research Laboratory (presently, the National Institute of Information and Communications and Technology), and worked on the VLBI (very long baseline interferometer) project at Kashima Space Research Center during 1985-1987. He moved to Hiraiso Space Environment Center in 1987 and worked on the space weather forecast project. He was assigned to be a director of Wakkanai Radio Observatory in 1991, and worked on ionospheric observations there until 1995. He moved to the Solar-Terrestrial Environment Laboratory (STEL), Nagoya University in 1995 to join the IPS (interplanetary scintillation) project. Since then, he has studied 3-dimensional structure

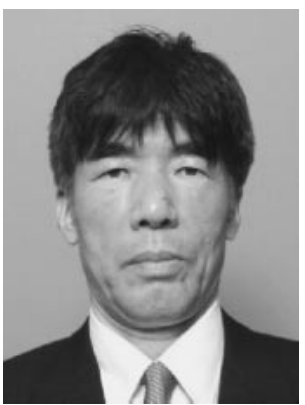
and dynamics of the solar wind using IPS observations. He was promoted Professor of STEL (chief of the IPS project) in 2008. He was awarded the Journal of Geomagnetism and Geoelectricity Award in 1993 and the Tanakadate Prize in 2011 from the Society of Geomagnetism and Earth, Planetary and Space Sciences. 